\title{
ABCs of the Bomber Problem and its Relatives
}

\author{
Richard Weber
}

\begin{abstract}
In a classic Markov decision problem of Derman, Lieberman, and Ross (1975) an investor has an initial capital $x$ from which to make investments, the opportunities for which occur randomly over time. An investment of size $y$ results in profit $P(y)$, and the aim is maximize the sum of the profits obtained within a given time $t$. The problem is similar to a groundwater management problem of Burt (1964), the notorious bomber problem of Klinger and Brown (1968), and types of fighter problems addressed by Weber (1985), Shepp et al (1991) and Bartroff et al (2010a). In all these problems, one is allocating successive portions of a limited resource, optimally allocating $y(x, t)$, as a function of remaining resource $x$ and remaining time $t$. For their investment problem, Derman et al (1975) proved that an optimal policy has three monotonicity properties: (A) $y(x, t)$ is nonincreasing in $t,(\mathrm{~B}) y(x, t)$ is nondecreasing in $x$, and $(\mathrm{C}) x-y(x, t)$ is nondecreasing in $x$. Theirs is the only problem of its type for which all three properties are known to be true.

In the bomber problem the status of (B) is unresolved. For the general fighter problem the status of $(A)$ is unresolved. We survey what is known about these exceedingly difficult problems. We show that $(\mathrm{A})$ and $(\mathrm{C})$ remain true in the bomber problem, but that (B) is false if we very slightly relax the assumptions of the usual model. We give other new results, counterexamples and conjectures for these problems.
\end{abstract}

Keywords Bomber problem · fighter problem · groundwater management problem · investment problem $\cdot$ Markov decision problem

\section{Stochastic sequential allocation problems}

\subsection{An investment problem}

In a classic Markov decision problem posed by Derman, Lieberman, and Ross (1975) an investor has initial capital of $x$ dollars, from which he can make withdrawals to fund investments. The opportunities for investment occur randomly as time proceeds. An investment of size $y$ results in profit $P(y)$, and the aim is maximize the sum of the profits obtained within a given time $t$. Without more ado let us set out this problem's dynamic programming equation. With an eye to variations of this problem to come, we make some notational changes. We take the remaining number of uninvested dollars to be discrete (a nonnegative integer) and denote it by $n(=0,1, \ldots)$ rather than $x$. The remaining time is also discrete and denoted by $t=0,1, \ldots$ We suppose that an investment opportunity occurs with probability $p(=1-q)$, independently at each remaining time step. We replace $P(y)$ with $a(k)$. Let $F(n, t)$ denote the expected total payoff from investments made over $t$

Richard Weber

Statistical Laboratory, University of Cambridge, Cambridge CB2 0WB, UK

E-mail: rrw1@cam.ac.uk 
further time steps, given initial capital $n$, and following an optimal policy. The dynamic programming equations is then

$$
F(n, t)=q F(n, t-1)+p \max _{k \in\{0,1, \ldots, n\}}[a(k)+F(n-k, t-1)],
$$

with $F(n, 0)=0$.

Let $k(n, t)$ denote a $k$ which is maximizing on the right hand side of $(1)$. We might have chosen to write $k^{*}(n, t)$, but it is less cluttering if we drop the star. Usually this $k$ is unique, but if it is not then we may define $k(n, t)$ to be the least maximizer. This is a mathematical nuisance, but it cannot affect our conclusions in any crucial way. Since time is discrete it is not hard to show that, given any positive integers $N$ and $T$, and assuming that $a(k)$ is strictly increasing, an infinitesimal perturbation of $p$ is sufficient to create a new problem in which the maximizer is unique for all $n \leq N$ and $t \leq T$.

Derman et al proved that $k(n, t)$ has three monotonicity properties, traditionally called (A), (B) and $(\mathrm{C})$. They can be proved to hold under the assumption that $a(k)$ is increasing and concave in $k$. The properties are

(A) $k(n, t)$ is nonincreasing $t$.

(B) $\quad k(n, t)$ is nondecreasing in $n$.

(C) $n-k(n, t)$ is nondecreasing in $n$.

A reader who is new to this problem should pause for a moment to reflect how highly reasonable and intuitive are the properties $(\mathrm{A}),(\mathrm{B})$ and $(\mathrm{C})$. Consider, for example, the case $p=1$. Then

$$
F(n, t)=\max _{\substack{k_{1}, \ldots, k_{t} \\ k_{1}+\cdots+k_{t} \leq n}}\left[a\left(k_{1}\right)+\cdots+a\left(k_{t}\right)\right],
$$

where $k_{i}$ dollars are allocated to the $i$ th investment. Assuming that $a(k)$ is a concave function, then it is clear that we should take all the $k_{i}$ as nearly equal as possible, so that $k_{1}=\lfloor n / t\rfloor$ and so trivially (A), (B), and (C) are all true.

So far, so unsurprising. What becomes surprising, is that there exist similar problems, with dynamic programming equations that are nearly the same as (1), for which the status of (A) and (B) remains a difficult open question.

In the remainder of this section we describe these similar problems. In Section 2 we discuss the discrete-time bomber problem. We present some counterexamples to show that (B) does not hold for some "near neighbours" of the bomber problem, and we discuss some ways that one might try to prove (B). Section 3 discusses some continuous-time problems. The paper wraps up with some discussion in Section 4. In Appendices A-C we place proofs of some new results (which are marked by superscripts in Table 1.) One purpose of this paper is to bring these problems to the attention of younger researchers who may not have met them before. So in Appendix A we include proofs of (A), (B), (C) for the investment problem in a streamlined style. The proofs of (A) and (B) are quite different from those given by Derman et al, and are somewhat easier because we take the resource to be composed of discrete units. The sense in which we prove (A) is much stronger. Our proof of (C) is new and establishes this property under more generous assumptions than in any previous work.

\subsection{A groundwater management problem}

The investment problem posed by Derman et al is very similar to a problem of groundwater management that dates back to Burt (1964). Interestingly, research in these two problem areas seems never to have intersected, or has their similarity been noticed previously. In the groundwater management problem the quantity of remaining resource equates to the level of water in an aquifer. Again, let us cut to the chase with a statement of the dynamic programming equation. Since the resource that we are now allocating is water, we denote its quantity by $x$, a continuous variable. We include a 
random variable, $R_{t}$, having some given distribution. This random variable models rainfall (runoff, or replenishment). Our dynamic programing equation is now

$$
F(x, t)=q \beta F(x, t-1)+p \max _{y \in[0, x]}\left[a(y)-c(x, y)+\beta E F\left(x-y+R_{t}, t-1\right)\right],
$$

with $F(x, 0)=0$. We have also introduced a factor $\beta(\in(0,1])$ to discount future rewards, and included a cost $c(x, y)$ for the effort of extracting water quantity of $y$, when the aquifer is at level $x$. It is usual to take $p=1$, but that is not necessary, so we have left it general. Knapp and Olson (1995) were the first to formulate conjectures (B) and (C) for this problem, but they were unable to prove them. Recently, Huh and Khrisnamurthy have discovered that this is because Knapp and Olsen adopted Burt's model of $c(x, y)=c(x) y$. They have shown that (B) and (C) may not hold under Burt's model, but that they do hold if one supposes instead that

$$
c(x, y)=\Gamma(x)-\Gamma(x-y),
$$

where $\Gamma(y)$ is increasing and concave in $y$ and $\Gamma(y+\delta)-\Gamma(y)$ is convex in $y$ for all positive $\delta$. For example, we might take $\Gamma(y)=1-e^{-y}$, so then $\Gamma(y+\delta)-\Gamma(y)=\left(1-e^{-\delta}\right) e^{-y}$. Or we could take

$$
c(x, y)=\int_{x-y}^{x} \gamma(z) d z,
$$

where $\gamma(z)$ is nonnegative, decreasing and convex. Although $F(x, t)$ need not be concave in $x$, the truth of (B) follows from showing that $F(x, t)+\Gamma(x)$ is concave in $x$. Collaborating further on this problem, (in Huh, Krishnamurthy, and Weber (2011)) we also have proved (A), (B) and (C), with arguments similar to those in Appendix A. We take $x$ to be a reference level, which may be be negative. But if we wish to constrain the stock of remaining ammunition or groundwater to be nonnegative then we can make this happen by letting $\Gamma(y)$ tend rapidly to $-\infty$ as $y$ decreases from 0 towards $-\infty$; this ensures that under an optimal policy the quantity of remaining resource (such as ammunition) will be maintained nonnegative.

\subsection{Fighter problems}

The profit function $a(y)$ may be given other interpretations. Let us look again at (1), but now narrate a story that a fighter aircraft is in flight for a remaining time $t$. During that time it will encounter enemy aircraft and try to shoot them down. An encounter with an enemy occurs with probability $p$ at each time step. If at such an encounter $k$ of $n$ remaining missiles are expended, that enemy plane will be shot down with probability $a(k)$. Now $F(n, t)$ is the expected number of enemy that will he shot down when following an optimal policy, starting with $n$ missiles and time $t$ to go.

Equation (1) assumes enemy aircraft are not dangerous. But perhaps they might be, in which case we might change the dynamic programming equation to

$$
F(n, t)=q_{t} F(n, t-1)+p_{t} \max _{k \in\{0,1, \ldots, n\}}[a(k)+c(k) F(n-k, t-1)],
$$

where now $c(k)$ is some nondecreasing function of $k$.

Notice that we have also added the wrinkle that $q_{t}=1-p_{t}$ is nonstationary, so that the probability of meeting an enemy at each discrete time step is known, but may vary with time. This generalization does not invalidate any known result for this, or for Derman et al's investment problem. Of course it makes more sense for $t$ to be continuous, but that is a limit of our discrete time model and is essentially the same.

In this generality, we must understand (A) correctly. Let us denote the least maximizer on the right hand side of $(3)$ by $k\left(n, p_{t-1}, \ldots, p_{1}\right)$. Then $(\mathrm{A})$ is to be understood as claiming that $k\left(n, p_{t-1}, p_{t-2}, \ldots, p_{1}\right) \leq k\left(n, 0, p_{t-2}, \ldots, p_{1}\right)$. This has been proved for the special cases $c(\cdot)=a(\cdot)$ and $c(\cdot)=1$. It is also very plausible that $k\left(n, p_{t-1}, p_{t-2}, \ldots, p_{1}\right) \leq k\left(n, 0, p_{t-2}, \ldots, p_{1}\right)$. This says that it is a property of an optimal policy that as the fighter continues flying forward in time, without 
yet meeting an enemy, the number of missiles that it should be ready to expend at the next meeting with the enemy is nondecreasing.

Generalizing this intuition even further, we may define a stronger version of property (A):

$(\mathrm{A})^{*} \quad k\left(n, p_{t-1}, \ldots, p_{1}\right)$ is nonincreasing in each of the variables $p_{t-1}, \ldots, p_{1}$.

In Appendix A we prove that $(\mathrm{A})^{*}$ holds in the special case of $(3)$ with $c(\cdot)=1$. We do not yet know whether or not it true for the special case $c(\cdot)=a(\cdot)$.

One very plausible model for $a(k)$ is that each missile fails to hit the enemy with probability $\theta$. If the enemy is not hit then it succeeds in destroying the fighter with some probability $v=1-u$. So with $\theta, v \in[0,1]$,

$$
\begin{aligned}
& a(k)=1-\theta^{k} \\
& c(k)=a(k)+(1-a(k)) u=u+(1-u) a(k)=1-v \theta^{k} .
\end{aligned}
$$

A convenient name for the above is the Bernoulli model. Let us emphasise that the decision to fire $k(n, t)$ units of ammunition must be made at the moment the enemy is met, and then the missiles are fired as a 'volley'. This is in contrast to the so-called 'shoot-look-shoot' manner, in which one can fire one missile, and then wait to see if that hits the enemy before deciding whether to fire another missile. Such problems are discussed by Sato (1997).

Bartroff, Goldstein, Rinott, and Samuel-Cahn (2010a), and Bartroff and Samuel-Cahn (2011) have coined the name 'frail fighter' for the case $u=0, c(\cdot)=a(\cdot)$, and the name 'invincible fighter' for the case $u=1$ and $c(\cdot)=1$.

The Invincible Fighter Problem corresponds to the investment problem of Derman et al and so (A), (B) and (C) all hold. Bartroff et al (2010a) have reproved these facts, using different styles of proof and in a continuous time setting.

A version of the Frail Fighter Problem, with $t=\infty$, was first considered by Weber (1985), who was motivated to pose the problem in an attempt to find an 'easier' bomber problem for which (B) might be proved true. However, (B) turned out to be false. E.g. for $\theta=1 / 2$, we found $k(4, \infty)=3$, but $k(5, \infty)=2$. An intuitive explanation for the failure of $(\mathrm{B})$ is that as the stock of ammunition increases from 4 to 5, we may anticipate being able to shoot down a greater number of the enemy, but to do this we must be more conservative in use of ammunition at the first meeting with the enemy.

Shepp, Simons, and Yao (1991) showed that this failure of (B) is not simply an artifact of the ammunition being discrete. They give a counterexample to (B) in which ammunition is continuous, (taking $\left.a(y)=c(y)=1-e^{-y}\right)$.

Both (A) and (C) are true for the frail fighter. (C) is very easy to prove. As observed above, (B) is not true. Bartroff and Samuel-Cahn (2011) have reported that the same counterexample that we gave to disprove (B) for $t=\infty$ is also a counterexample for $t>2.694 \cdots$. They have also proved (A). That proof is not obvious, but essentially involves showing that $\log (1+F(n, t))$ is supermodular in $(n, t)$ (or to put it another way that $(1+F(n, t)) /(1+F(n-1, t))$ is nondecreasing in $t)$. The arguments are very similar to those that were used by Samuel (1970) to prove (A) for the bomber problem. However, this is rather different to the (easier) proof of $(\mathrm{A})$ for the invincible fighter which involves showing supermodularity of $F(n, t)$.

In Appendix A we prove two new results. The first of these is that $(\mathrm{A})^{*}$ holds for the invincible fighter. The second is that (C) holds for the general fighter under the simple conditions that $a(k)$ is increasing and concave, and $c(k)$ is increasing and log-concave. It is not necessary to assume the special form $c(k)=u+(1-u) a(k)$.

Open Problem for the Fighter It is not known whether (A) holds for the general fighter and the Bernoulli model with $0<u<1$. We know that (A) holds for $u=0$ and $u=1$. However, as mentioned above, the proofs of (A) are quite different for these two cases and there is no obvious way to interpolate between them.

If $(\mathrm{A})$ is true for arbitrary $u$ then it should be true in the very special case that $p_{t}=1$ for all $t$. This means that starting in state $(n, t)$ we know that the enemy will surely be met exactly $t$ times. 
The proof for this case is not as easy as one might think! A proof is in Appendix B. We would be interested to see a simpler proof.

\subsection{Bomber Problem}

Finally, we come to the notorious bomber problem, which was posed long ago by Klinger and Brown (1968). This is the problem that we obtain from (3) on putting $a(k)=0$ and changing the boundary condition to $F(n, 0)=1$. This makes $F(n, t)$ the probability of successfully defending a bomber against attacking enemy planes that it meets on the way to its target, starting in state $(n, t)$ and using an optimal ammunition rationing policy. Klinger and Brown proved $(\mathrm{C})$ and showed that the truth of (B) would imply (A). A new proof of (C) is in Appendix A. The proof of (A) (not assuming (B)) is difficult and is due to Samuel (1970). Her proof was recast in discrete time by Simons and Yao (1990), who proved (A) by showing that $k\left(n, p_{t-1}, \ldots, p_{1}\right)$ is nondecreasing in $p_{t-1}$. They made the conjecture that $(\mathrm{A})^{*}$ holds for the bomber problem, but could not prove it. As mentioned above, we have proved $(\mathrm{A})^{*}$ for the invincible fighter.

Open Problem for the Bomber Despite 40 years of research, it is still not known if (B) is true for the bomber problem. So far as I know, the best we can say about (B) is that $k(n+1, t) \geq k(n, t)$ if either $n \leq 3$ or $t \leq 3$, and also that $k(n, t)=1 \Longrightarrow k(n-1, t)=1$ for all $t$. These results are recorded in Appendix C.

\subsection{A table of results and open problems}

We summarise in Table 1 the status of (A), (B) and (C) for the set of problems discussed above, all of which are defined by cases of dynamic programming equation (3). The proofs of various results marked with a 'yes' can be established using properties of functions that are supermodular, concave, and/or totally positive. The important result of Topkis (1978) about the monotonicity of the maximizer of a supermodular functions is often useful, and this is essentially what is being done in the proof of (A) in Appendix A. It is easy to prove (C) for the bomber problem or invincible fighter. This needs just a few (though slightly different) lines of algebra in each case, and it holds under very weak conditions. Proofs for (A) and (B) are substantially more difficult. Results which we believe to be new in this paper are given a superscript.

The reader should appreciate that it is of no practical value to military planners to resolve the open problems marked by ? in Table 1. If (B) is not true for the bomber problem, but we were to restrict ourselves to the class of policies for which (B) holds, then we would see only a miniscule reduction in the probability of successfully surviving until time $t$. The operations research literature is full of papers in which it is proved that an optimal policy (in some given problem) has the character of changing monotonically in some manner as some state or parameter of the problem varies, e.g. that the optimal rate at which to serve a queue is nondecreasing in the number of customers waiting. By and large, such results are both obvious and easy to prove. What makes the bomber problem so intriguing is that (B) is obvious, but it is very far from being easy to prove or disprove. Apart from the sheer intellectual challenge that it poses, one imagines that a resolution of (B) for the bomber problem might lead to interesting new theoretical ideas, and new techniques with wider applicability.

\section{So what do we make of (B) for the bomber problem?}

The doubly discrete time bomber problem arises from (3) upon setting $a=0$ and $F(n, 0)=1$. For this problem, in which both ammunition and time are discrete, Simons and Yao (1990) have shown that (A) and (C) hold under the assumption that $c(k)=1-v \theta^{k}$. Their proofs remain valid for any $c(k)$ that is nondecreasing and $\log$-concave in $k$ (i.e. such that $\log c(k)$ is concave), and with 
Table 1 Known results and open problems for fighter and bomber problems that are doubly discrete (in both ammunition and time) as defined by dynamic programming equation (3). Question marks indicate the two exceedingly difficult open problems. Results which we believe are new in this paper have a superscript and are located within the text as follows: [1] Appendix B; [2] Appendix A.2 (the strengthened version of (A)); [3] Counterexample 1; [4] Counterexample 2; [5] Counterexamples 3 and 4; [6] Appendix A.3. Other results have superscripts amongst the set $\{b, d, g, s, S, y, w\}$, shown here and after dates of papers listed amongst the references. These are attached with some license, as the referenced paper may have addressed a model with continuous ammunition or time, or it may have proved something weaker, but one can see that the stronger result could have been proved by the same method. Superscript are omitted if something stronger exists in another row of the table.

\begin{tabular}{|c|c|c|c|c|c|c|c|}
\hline$F(n, 0)$ & $a(k)$ & $c(k)$ & $p_{t}$ & (A) & (B) & $(\mathrm{C})$ & \\
\hline 0 & Bernoulli & $u+(1-u) a(k)$ & $p$ & $?$ & $\mathrm{no}^{[w, s, b, g]}$ & & general fighter \\
\hline 0 & concave & $u+(1-u) a(k)$ & 1 & yes $^{[1]}$ & & yes & general fighter \\
\hline 0 & concave & $\delta$ & & yes $^{[2, d, b]}$ & $\operatorname{yes}^{[d, b, g]}$ & $\operatorname{yes}^{[d, b, g]}$ & invincible fighter \\
\hline 0 & concave & $a(k)$ & & yes $^{[b, g]}$ & no & yes & frail fighter \\
\hline 1 & 0 & Bernoulli & $p$ & yes & $?$ & yes & bomber \\
\hline 1 & 0 & log-concave & 1 & yes & yes & yes & bomber \\
\hline 1 & 0 & log-concave & & yes $^{[S, y, g]}$ & $\mathrm{no}^{[3]}$ & yes $^{[S, g]}$ & bomber \\
\hline 1 & 0 & log-concave & $p$ & yes & $\mathrm{no}^{[3]}$ & yes & bomber \\
\hline 1 & 0 & concave & $p$ & yes & $\mathrm{no}^{[4]}$ & yes & bomber \\
\hline concave & 0 & Bernoulli & $p$ & yes & $\mathrm{no}^{[5]}$ & yes & bomber \\
\hline$\geq 0$ & concave & log-concave & & & & yes $^{[6]}$ & bomber/fighter \\
\hline
\end{tabular}

nonstationary meeting probabilities, $p_{t}$ as written in (3). It is intuitive that this result implies that $\log$-concavity of $c(k)$ is sufficient for $(\mathrm{A})$ and $(\mathrm{C})$ to hold in other versions of the problem, in which either ammunition is discrete and time is continuous (as previously shown by Samuel (1970)), or in the doubly continuous version (as shown directly in Bartroff, Goldstein, Rinott, and Samuel-Cahn (2010a)). However, we say 'intuitive' because the arguments that are needed to pass from discrete to continuous models are not trivial. Indeed, a proper discussion of this issue could probably fill an entire paper.

The observation that $(\mathrm{A})$ and $(\mathrm{C})$ hold if $c(k)$ is log-concave, and $p_{t}$ nonstationary, raises the following obvious question.

Might (B) hold under assumptions just as weak as those for which (A) and (C) hold?

The answer is no, as the following counterexamples demonstrate. Notice that if $c(k)$ is an increasing and concave function of $k$ then it is also log-concave in $k$. For this reason Counterexample 1 shows nothing stronger that does Counterexample 2. However, we include it because it is a much simpler example to construct. For both counterexamples we assume $p_{t}$ is stationary, i.e. $p_{t}=p$, for some $p$.

Counterexample 1 There exists a nondecreasing and log-concave $c(k)$ for which (B) does not hold.

Let $p_{t}=10 / 11$ for all $t$, and $\{c(0), c(1), c(2), c(3), c(4), \ldots\}=\left\{0, \frac{3}{16}, \frac{1}{2}, 1,1, \ldots\right\}$. Note that $c(i)^{2} \geq$ $c(i+1) c(i-1)$ for all $i \geq 1$, so $c(\cdot)$ is log-concave.

Then $\{k(n, 4)\}_{n=1,2, \ldots}=\{1,1,1,2,2,3,2, \ldots\}$, i.e. $3=k(6,4)>k(7,4)=2$.

Counterexample 1 was easily found by trial and error. However, it is not as easy to find a failure of (B) for a concave $c(k)$. The next counterexample explains how that may be done.

Counterexample 2 There exists a nondecreasing and concave $c(k)$ for which (B) does not hold. 
The construction of this counterexample is based on the idea that if ammunition is continuous then (B) can hold only if $F(x, t)$ is log-concave in $x$. So let $x$ denote a continuous quanitity of ammunition, and observe that

$$
\begin{aligned}
& F(x, 1)=q+p c(x) \\
& F(x, 2)=\max _{y \in[0, x]}\left[q^{2}+p q c(y)+q p c(x)+p^{2} c(y) c(x-y)\right] .
\end{aligned}
$$

Our counterexample is constructed by cooking up a nondecreasing and concave function $c(\cdot)$ for which $F(x, 2)$ is not log-concave at a particular value of $x$. Let us do this at $x=3$. We design $c(\cdot)$ so that

(i) $c(\cdot)$ is nondecreasing and concave;

(ii) $F(x, 2)$ is not log-concave at $x=3$;

(iii) $y(x, 2)=\arg \max _{y \in[0, x]}[c(y) F(x-y, 1)]=\frac{1}{2}(1+x)$ in the neighbourhood of $x=3$.

Condition (iii) is arbitrary, but is imposed so that we have an easy way to figure out the value of $F(x, 2)$ in the neighbourhood of $x=3$, i.e.

$$
F(x, 2)=q F(x, 1)+p c\left(\frac{x+1}{2}\right) F\left(\frac{x-1}{2}, 1\right) .
$$

Note that $y(3,2)=2$ and $3-y(3,2)=1$. This means that conditions equivalent to (i)-(iii) can be expressed in terms of just nine variables: $\left\{c(x), c^{\prime}(x), c^{\prime \prime}(x)\right\}$, at $x=1,2,3$. (To save space, we omit writing down these conditions; it is straightforward.) By using Mathematica's FindInstance function and then doing some judicious hand-tweaking, it turns out that we can achieve (i)-(iii) by taking $p=2 / 3$, and $\{c(1), c(2), c(3)\}=\{1 / 4,7 / 16,1 / 2\},\left\{c^{\prime}(1), c^{\prime}(2), c^{\prime}(3)\right\}=\{1 / 4,7 / 48,1 / 64\}$, $\left\{c^{\prime \prime}(1), c^{\prime \prime}(2), c^{\prime \prime}(3)\right\}=\{0,0,0\}$, and making $c$ linear in intervals. It is now convenient to rescale by a factor of 6 , so that $1,2,3$ become $6,12,18$. By this means we arrive at

$$
c(x)=\min \left\{\frac{1}{24} x, \frac{7}{48}+\frac{7}{288} x, \frac{371}{1152}+\frac{49}{4320} x, \frac{29}{64}+\frac{1}{384} x, 1\right\} .
$$

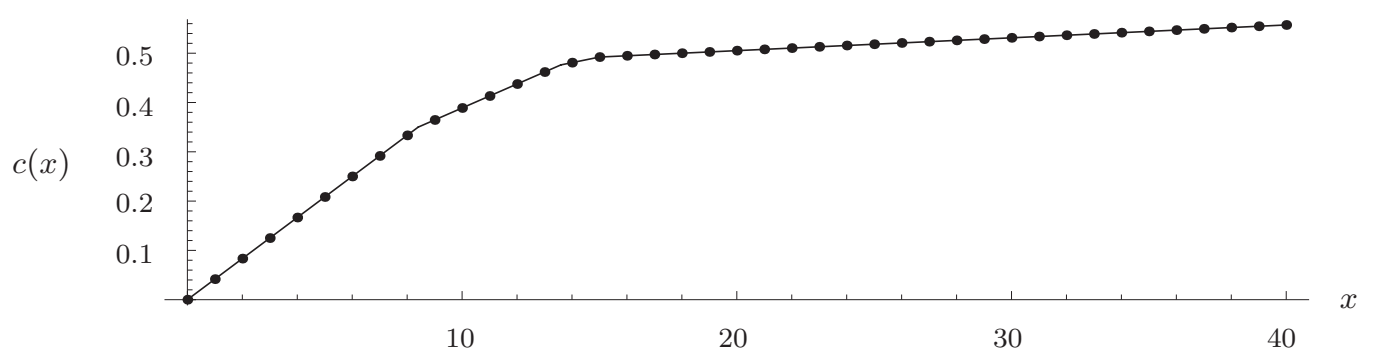

Fig. 1 A concave $c(\cdot)$ for which (B) does not hold in the bomber problem, when $p=2 / 3$, ammunition is either continuous or discrete, and time is discrete.

Clearly $c(x)$ is a concave function of $x$ (as it is the minimum of linear functions of $x$ ). We find, in contradiction to $(\mathrm{B})$,

$$
\begin{aligned}
& y(32,3)=\arg \max _{y \in[0,5.24]}[c(y) F(31.4-y, 2)]=14.0079 \\
& y(33,3)=\arg \max _{y \in[0,5.25]}[c(y) F(31.5-y, 2)]=13.9174 .
\end{aligned}
$$

Note that $32-y(32,3)=32-14.0079=17.9921$ and $33-y(33,3)=33-13.9174=19.0826$ lie in the neighbourhood of $x=3 \times 6=18$, where we have ensured that $F(x, 2)$ is not log-concave. This is what leads to (B) failing to be true. 
This same example also shows that (B) fails when ammunition is discrete. It is easy to calculate that

$$
\begin{aligned}
&\{k(n, 2)\}_{n=1}^{40}=\{1,2,3,4,5,6,7,8,9,9,9,9,10,10,11,11,12,12,13,13 \\
&13,14,14,14,14,14,14,15,15,15,16,17,18,19,20,21,22,23,24,25\} \\
&\{k(n, 3)\}_{n=1}^{40}=\{1,2,3,4,5,6,6,7,7,8,8,8,8,9,9,9,9,10,11,12,13,13,13,13,14,14,14,14,14,14,15,14,15,14,14,15,15,15,15,15\} .
\end{aligned}
$$

So $k(31,3)=15>14=k(32,3)$, in contradiction to $(\mathrm{B})$.

It is interesting that within the definition of $c(k)$ in (4) the term for which the minimum is achieved in (4) is the $i(k)$ th, where $\{i(k)\}_{k=1}^{20}=\{1,1,1,1,1,1,1,1,2,2,2,2,2,3,\{3,4\}, 4,4,4,4,4\}$. So the third term within the minimum on the right hand side of (4) is active in defining $c(k)$ only for $k=14$ (and third and fourth terms are equal at $k=15$ ). However, this is crucial, for if we alter $c(\cdot)$ by omitting this term then $k(n, 3)$ is monotone in $n$.

2.1 How might we prove (B) in the Bernoulli case?

Counterexample 2 shows us that $(\mathrm{B})$ is not true unless we make some strong assumption about $c(k)$. In particular, the status of $(\mathrm{B})$ for the Bernoulli case of $c(k)=1-\theta^{k}$ remains open. Researchers have tried many things in pursuit of a proof of $(B)$ for this case, or of finding a counterexample. It is worth recording some dead ends, if only to save others their rediscovery.

Might we vary the last missile's effectiveness? Take the Bernoulli model and suppose that the last unit of ammunition has a miss probability $\psi \in[\theta, 1]$, so that $c(k)=1-\psi \theta^{k-1}$ when the last missile is fired amongst a set of $k$. We might conjecture that $k(n, t, \psi)$ is nonincreasing as $\psi$ increases from $\theta$ to 1 . If this is true, then (B) would follow from $k(n, t)=k(n, t, \theta) \geq k(n, t, 1)=k(n-1, t)$. No counterexample to this conjecture has been found.

A somewhat similar trick would be to change the boundary condition to $F(0,0)=1$ and $F(n, 0)=$ $1+\lambda, n \geq 1$. As $\lambda$ increases this would impose a constraint that a last missile be kept unused. Now letting $k(n, t, \lambda)$ denote the optimal units of ammunition to fire we would have $k(n, t, 0) \geq k(n, t, \infty)=$ $k(n-1, t)$. But for this we have Counterexample 3, in which $k(n, t, \lambda)$ increases, and then decreases, as $\lambda$ increases.

Counterexample 3 Suppose that $p=\theta=3 / 5, F(0,0)=1$ and $F(n, 0)=1+\lambda, n \geq 1$. Let $k(n, t, \lambda)$ denote the optimal number of missiles to fire. Then $k(8,3,0.4)=3, k(8,3,0.6)=4$ and $k(8,3,0.8)=3$.

This even gives a counterexample to $(\mathrm{B})$ when the terminal rewards are those given by a constant $\lambda, \lambda=0.6$. We find $k(8,3,0.6)=4$, and $k(9,3,0.6)=3$.

One could vary $F(n, 0)$ in other ways. Interestingly, $(\mathrm{B})$ appears to be true when $F(n, 0)=n$, i.e. when the aim is to maximize the expected number of missiles that are delivered to the destination.

Might we prove (B) by value iteratation with the dynamic programming equation? An iterative argument with the dynamic programming equation lies at the heart of the proof of almost every one of the positive results in Table 1. One might have hoped that the same approach would work for (B) if only an appropriate inductive hypothesis could be formulated. Suppose that one takes the dynamic programming equation of

$$
F(n, t)=q^{t}+\sum_{s=1}^{t} q^{s-1} p \max _{k}[c(k) F(n-k, t-s)]
$$

and tries to inductively show some property of $F(n, t)$, from which then (B) might follow, by using iterates of the form

$$
F_{j+1}(n, t)=q^{t}+\sum_{s=1}^{t} q^{s-1} p \max _{k}\left[c(k) F_{j}(n-k, t-s)\right], \quad j=0,1, \ldots,
$$


starting with $F_{0}=1$, say. Then (5) is the dynamic programming equation for a problem of maximizing the probability of surviving until either time $t$ has elapsed or there have been $j$ meetings with the enemy. Let $k_{j}(n, t)$ denote the maximizer on the right hand side of (5). Counterexample 4 shows that $(\mathrm{B})$ does not hold in respect of $k_{j}(n, t)$.

Counterexample 4 Consider the Bernoulli model with $p_{t}=1 / 2$ and $c(k)=1-(3 / 5)^{k}$. We find $k_{8}(7,18)=2$, but $k_{8}(8,18)=1$, so $k_{j}(n, t)$ is not nondecreasing in $n$.

Note also, the somewhat surprising fact that $k_{7}(7,17)=1$ and $k_{8}(7,17)=2$, so $k_{j}(n, t)$ can be increasing in $j$. (More usually it is nonincreasing.)

Might we prove that $F(n, t)$ is log-concave in $n$ ? Almost the first thing that any researcher appreciates when he or she begins to consider the question of whether (B) does or does not hold for the bomber problem is this: that a sufficient (but not necessary) condition for (B) is that $\log F(n, t)$ is concave in $n$. This is because the condition of log-concavity is equivalent to saying that $F(n, t) / F(n-$ $1, t)$ is nonincreasing in $n$. This would imply that the inequality $c(k+1) F(n-k-1, t) \geq c(k) F(n-k, t)$ would remain true as $n$ increases beyond the least value for which it is true, and this fact would imply (B). However, $F(n, t)$ is not log-concave (at least not in the discrete-time discrete-ammunition bomber problem). A failure of log-concavity means that for some $n$ and $t$,

$$
\Delta(n, t):=\frac{F(n+1, t)}{F(n, t)}-\frac{F(n, t)}{F(n-1, t)}>0 .
$$

Weber (1985) gave the example $(p, \theta)=(0.64,0.65)$ with $\Delta(14,3)>0$. Simons and Yao $(1990)$ gave the example $(p, \theta)=(0.58,0.6), \Delta(8,3)=0.0001402$. For $(p, \theta)=(0.7015,0.72428)$ we can obtain an even greater value of $\Delta(8,3)=0.0004779$. This is the largest positive value of $\Delta(n, t)$ that I have found for any choice of the parameters $(n, t, p, \theta)$.

Lest one think that a failure of log-concavity arises only when $t$ or $n$ is small, or when $p$ is large, let us note that there are examples of non-log-concavity even in the following cases. For $(p, \theta)=(0.1,0.01000048), \Delta(8,3)=4.58768 \times 10^{-15}$ (a number that really is positive, since we can also express it as an exact fraction). One can also find $\Delta(n, t)>0$ for arbitrarily large $t$, by taking $n$ large, and $p$ and $\theta$ both tending to 1 . It can even happen that log-concavity fails infinitely often. For $(p, \theta)=(0.99,0.99)$, we find $\Delta(n, 3)>0$ for all $n=16,22,28,34,40, \ldots$, i.e. for an infinite number of $n$.

The values of $k(n, t), n \leq 9$ and $t \leq 3$, are the same in many of the examples mentioned above. But these examples are also very sensitive to the parameters. A slight change in $p$ or $\theta$ makes $\Delta(8,3) \leq 0$. Figure 2 shows a plot of $-F(8,3) \Delta(8,3)$ for all values of $p$ and $\theta$ in the interval $[0,1]$. Log-concavity fails when this quantity is negative, which occurs, within a small trench where $p$ is a bit less than $\theta$. We have not found any non-log-concavity for $p$ approaching 0 while $\theta$ is bounded away from 0 . This is the appropriate approximation of a continuous-time model, for which no instances of nonlog-concavity have been found. Of course (B) is has never been found to be false in the discrete-time setting, despite very numerous calculations and intelligently-directed searches for a counterexample.

In concluding this section, we remark that in Table 1 we have not considered the possibility that the functions $a(k)$ and $c(k)$ might depend on time. Indeed, (C) remains true under such a relaxation in assumptions. However, because of a failure of log-concavity (B) is definitely not true in the bomber problem if $c(k)$ is allowed to depend on time.

It can be proved that $F(n, t)$ is log-concave for $t=1,2$ (see Appendix $\mathrm{C}$ ).

In the doubly-continuous problem bomber problem, $(\mathrm{B})$ can only be true if $\log P(x, t)$ is concave in $x$ (see Weber (1985) and Simons and Yao (1990)).

\section{Continuous-time bomber and fighter problems}

It is also interesting to look at versions of these problem in which either one or both of 'remaining time' or 'remaining ammunition' is taken to be a continuous variable. By making a variable continuous 


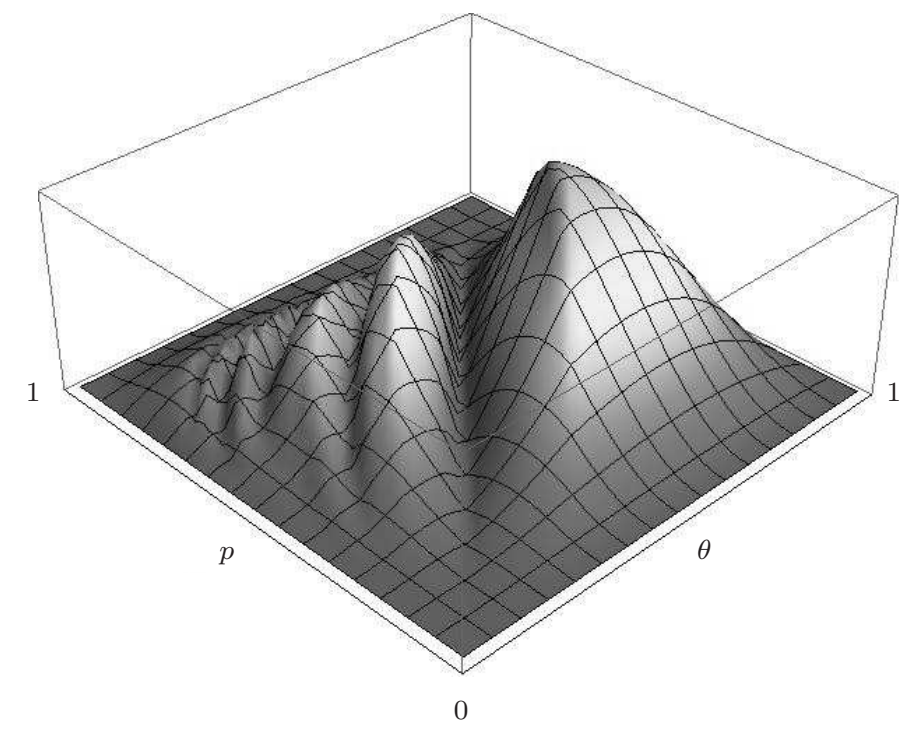

Fig. $2 F(8,3)^{2}-F(7,3) F(9,3)$ as a function of $p$ and $\theta$. The region of non-log-concavity, where this quantity is negative, lies in the central trench, where $p$ is a bit less than $\theta$.

a proof of (A) or (B) should be easier (since, as we have remarked above, intutitively a result for a discrete variable implies the same result in its continuous analogue). For example, making time continuous, the bomber problem has a defining equation, for $n \geq 1$, of

$$
F(n, t)=e^{-t}+\int_{0}^{t} \max _{k \in\{1, \ldots, n\}}[c(k) F(n-k, s)] e^{-(t-s)} d s .
$$

with $F(0, t)=e^{-t}$ and $F(n, 0)=1$. It is attractive to rewrite this, by defining $G(n, t)=e^{t} F(n, t)$. Then we have the apparently very simple recursion, for $n \geq 1$,

$$
G(n, t)=1+\int_{0}^{t} \max _{k \in\{1, \ldots, n\}}[c(k) G(n-k, s)] d s .
$$

with $G(0, t)=G(n, 0)=1$. No example has been found in which $F(n, t)$ is not log-concave in $n$. However, proving it from (6) or (7) seems difficult, since the behaviour of a measure of log-concavity is not at all well-behaved. We see this in Figures 3 and 4.

We can show that $(\mathrm{B})$ is true in any problem in which we are contrained (or it is optimal) never to fire more than 2 missiles. An example is when $c(k)=1-\theta^{\min (2, k)}$.

Note first that $k(n, t) \geq k(n-k(n, t), t)$. If this were not true, then by (A), we would have $k(n, t)<j(s):=k(n-k(n, t), s)$, for all $s \leq t$, and so

$$
c(k(n, t)) G(n-k(n, t), t)=c(k(n, t))\left[1+\int_{0}^{t} c(j) G(n-k(n, t)-j(s), s) d s\right] .
$$

The right hand side can be made greater simply by increasing $k(n, t)$ by 1 and decreasing $j(s)$ by 1 . From this fact we can deduce $(\mathrm{B})$, because $k(n, t)=1$ implies $k(n-k(n, t), t)=k(n-1, t) \leq 1$.

Suppose that we take $c(1)=1 / 2, c(k)=1, k \geq 2$. This is a concave (but not Bernoulli) choice of $c(\cdot)$. The above argument establishes that $(\mathrm{B})$ is true, but it turns out that $F(n, t)$ is not log-concave. Thus log-concavity is not necessary for $(\mathrm{B})$. Similarly, suppose $c(k)=1-(7 / 8)^{\min (3, k)}$. It is clear 


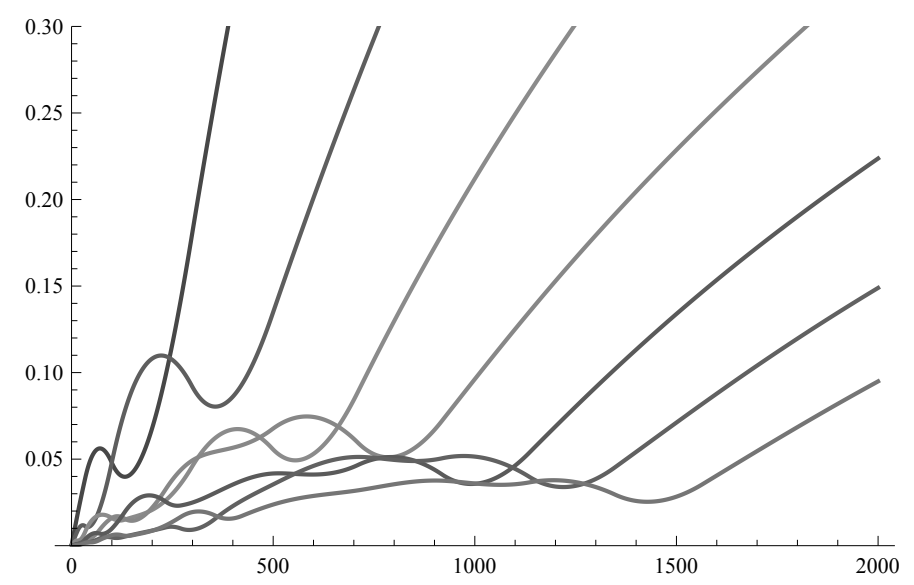

Fig. $3 F(n, t) / F(n-1, t)-F(n+1, t) / F(n, t)$, for the continuous-time bomber problem with $\theta=1 / 2$, for $0 \leq t \leq 20$ and $n=2, \ldots, 8$ (reading left to right across the asymptotes). Although we see that $F(n, t)$ is log-concave in $n$, the fact that these functions are not monotone increasing, in either $n$ or $t$, means that it is probably difficult to prove that $F(n, t)$ is log-concave in $n$ by some sort of induction on $n$, or using differential equations in $t$.

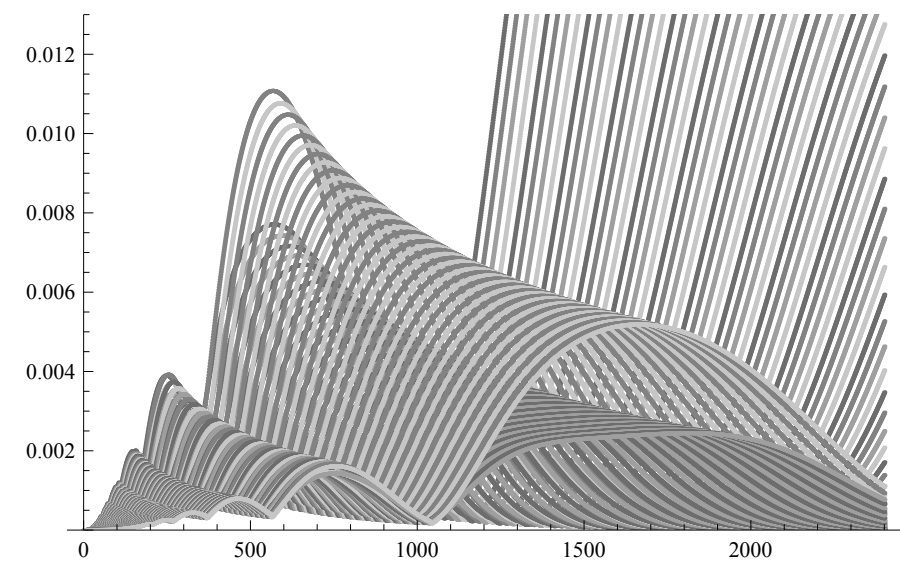

Fig. 4 This is a plot of $-\Delta(n, t)=F(n, t) / F(n-1, t)-F(n+1, t) / F(n, t)$, for the discrete-time bomber problem, with $(p, \theta)=(1 / 10,1 / 2)$, for $1 \leq t \leq 2400$ and $n=60, \ldots, 99$ (reading left to right across the asymptotes). Notice that the behavior differs depending on whether $n$ is even or odd. One continues to see essentially the same sort of thing when making $p$ even smaller, approaching even more closely the continuous time model. In this example $-\Delta(n, t)$ becomes very close to zero around $t=2000$ but never actually goes negative.

that an optimal policy need never fire more than 3 missiles. For this model it turns out that $F(n, t)$ is not log-concave, but calculations indicate that (B) is true.

One can also imagine making the quantity of ammunition a continuous variable, as indeed is appropriate in the groundwater management problem. As remarked above, if (B) is true with continuous ammunition then $F(x, t)$ must be log-concave in $x$.

A spend-it-all region for the bomber problem in the case of continuous ammunition and continuous time is addressed by Bartroff, Goldstein, and Samuel-Cahn (2010b), and Bartroff (2011). In Appendix $\mathrm{C}$ we describe the spend-it-all region in the doubly discrete setting.

So far as I know, there is no problem for which a re-framing in continuous variables changes the known truth status of any of (A), (B) or (C). 


\section{Where next?}

Over the course of the past 40 years the increasing power of computers and computational software has provided a motivation for returning to the bomber problem in the hope of making new discoveries. To some extent that has paid off, since we have now discovered the fact that (B) relies crucially on the Bernoulli model for $c(k)$.

In this paper we have focused upon the doubly-discrete bomber problem, in which both remaining ammunition and time are nonnegative integers. It makes sense to look at this setting of the problem because if (B) is true in this setting then it is also true if either time or ammunition is taken to be continuous, and if (B) is false it may be easiest to find a counterexample in this setting. Arguments can still be made for (B) being true or false.

In favour of (B) being true is the fact that despite a truely enormous amount of computational experimentation no counterexample has been found when the model is $c(k)=1-\theta^{k}$. Such computation has not been conducted simply by trying random parameter values, but has been intelligently-focused in areas of the parameter space $(\theta, p) \in[0,1] \times[0,1]$ where $P(n, t)$ fails most dramatically to be logconcave in $n$, as indeed must be the case if $(\mathrm{B})$ is false. Today's computing power means that one can compute $k(n, t)$ for values of $n$ and $t$ numbering in thousands, and that for smaller $n, t$ we can even optimize carefully by varying $\theta$ and $p$. None of this exploration has produced a counterexample to (B). Notice that Counterxamples 3, 4, 5 have been found for quite small values of $n$ and $t$. There has been no need to take these particuarly large.

In favour of (B) being false is the fact that (B) is false for some very "near-neighbours" of the bomber problem, such as problems that are obtained by varying $c(k)$ away from the Bernoulli model, or by modestly changing the boundary condition of $F(n, 0)=1$. If one thinks that (B) is intuitively true, why does this intuition fail for those near-neighbours for which (B) is false? Recall that (B) does not hold in the frail fighter problem. In Section 1.3 we gave an intuitive argument why this should be so. But this begs the opposite question: why does not the same intuitive argument convince us that (B) is false for the invincible fighter, for which (B) is provably true?

Here are some research tasks that readers might like to pursue.

(i) Construct a nice proof that in the case $c(k)=1-\theta^{k}$,

$$
P(n, 2)=q^{2}+q p c(n)+p \max _{k}[q c(k)+p c(k) c(n-k)]
$$

is a log-concave function of $n$. I can prove this result, but not in a manner that is sufficiently attractive to reproduce here. This result implies that $k(n, 3)$ is nondecreasing in $n$.

(ii) Let $c(k)=1-\theta^{k}$. Pick some particular value for $\theta$, say $\theta=1 / 2$. Show that despite the fact that $P(n, 3)$ is not necessarily log-concave $n$, we do have (B), i.e. that $k(n, 4)$ is nondecreasing in $n$.

(iii) For $t=3,4$, show that there exists $\bar{p}_{t}$ such that $P(n, t)$ is log-concave in $n$ for all $p \leq \bar{p}_{t}$. Evaluate the extent to which $\bar{p}_{4}$ must be taken smaller than $\bar{p}_{3}$.

(iv) Examine more carefully Counterexample 2. Explore the boundaries of this counterexample. How small is it possible to make $p$ ? Can one find $P(n, t)$ failing to be log-concave, when $c(k)$ is an arbitrary concave function and we set the problem in continuous time?

(v) We know (B) is true for the special model $c(k)=1-\theta^{\min (2, k)}$. What if $c(k)=1-\theta^{\min (3, k)}$ ?

Acknowledgements I thank Sheldon Ross for introducing me to the bomber problem (in 1983-84). I am grateful to Tim Huh and Chandra Krishnamurty for alerting me to the similar strand of work on groundwater management problems. This paper was assembled while preparing a talk for the Third International Workshop on Sequential Methods (Stanford, June 14-16, 2011). At this workshop I very much enjoyed meeting others who have also been fascinated by, and contributed to, the study of ABCs of the bomber problem and its relatives: Jay Bartroff, Larry Goldstein (who organised our session), Ester Samuel-Cahn, Larry Shepp and Yi-Ching Yao. Their comments on drafts of this paper have been helpful. 


\section{References}

Bartroff J (2011) A proof of the bomber problem's spend-it-all conjecture. Sequential Analysis 30:52-57

Bartroff J, Samuel-Cahn E (2011) ${ }^{[b]}$ The fighter problem: optimal allocation of a discrete commodity. Adv Appl Probab 43:121-130

Bartroff J, Goldstein L, Rinott R, Samuel-Cahn E (2010a) $[g]$ On optimal allocation of a continuous resource using an iterative approach and total positivity. Adv Appl Probab 42(3):795-815

Bartroff J, Goldstein L, Samuel-Cahn E (2010b) The spend-it-all region and small time results for the continuous bomber problem. Sequential Analysis 29:275-291

Burt OR (1964) Optimal resource use over time with an application to ground water. Manage Sci 11(1):80-93

Derman C, Lieberman GJ, Ross SM (1975) ${ }^{[d]}$ A stochastic sequential allocation model. Oper Res 23(6):1120-1130

Huh WT, Krishnamurthy CK, Weber RR (2011) Concavity and monotonicity properties in a groundwater management model, to appear in Naval Res. Logist.

Klinger A, Brown TA (1968) Allocating unreliable units to random demands. In: Karreman H (ed) Stochastic Optimization and Control, Wiley, pp 173-209

Knapp KC, Olson LJ (1995) The economics of conjunctive groundwater management with stochastic surface supplies. Journal of Environmental Economics and Management 28(3):340-356

Marshall AW, Olkin I (1979) Inequalities: Theory of Majorization and Its Applications. Academic Press, New York

Samuel E (1970) ${ }^{[S]}$ On some problems in operations research. J Appl Probab 7:157-164

Sato M (1997) A stochastic sequential allocation problem where the resources can be replenished. J Oper Res Soc Japan 40(2):206-219

Shepp LA, Simons G, Yao YC (1991) ${ }^{[s]}$ On a problem of ammunition rationing. Adv Appl Prob 23:624-641

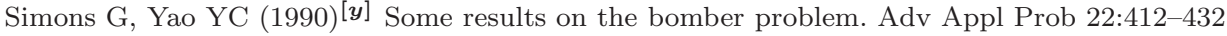

Topkis DM (1978) Minimizing a submodular function on a lattice. Oper Res 26(2):305-321

Weber RR (1985) ${ }^{[w]}$ A problem of ammunition rationing. In: Radermacher FJ, Ritter G, Ross SM (eds) Conference report: Stochastic Dynamic Optimization and Applications in Scheduling and Related Fields, held at University of Passau, Facultät für Mathematik und Informatik, p 148

\section{A Proofs of $(\mathrm{A})^{*},(\mathrm{~B})$ and (C) for Derman, Lieberman, and Ross's investment problem}

We sketch (for discrete time and discrete resource) proofs of $(A)^{*},(B)$ and $(C)$ for the investment problem of Derman, Lieberman, and Ross (1975) and also, where it is simple to do so, some stronger results. The ordering below is pedagogical, rather than alphabetical. Throughout we assume that $a(k)$ and $c(k)$ are nondecreasing in $k$ and, respectively, concave and log-concave.

\section{A.1 Proof of $(B)$}

This is for the problem dynamic programming equation of the investment problem/invincible fighter. It is the special case of (3) in which we take $c(\cdot)=1$.

As we shortly explain below, (B) is true if $F(n, t)$ can be shown to be concave in $n$. That is, for all $n, t$,

$$
F(n+2, t)-2 F(n+1, t)+F(n, t) \leq 0 .
$$

Suppose that (8) is true. Recall that $k(n, t)$ is the least $k$ for which $a(k)+F(n-k, t-1)$ is maximal. Suppose that $k_{1}=k(n, t)$ and $k_{2}=k(n+1, t)$, and that in contradiction to (B) we have $k_{2}<k_{1}$. By interchanging the usages of $k_{1}$ and $k_{2}$, so that $k_{2}$ is used in states $(n, t)$ and $k_{1}$ is used in state $(n+1, t)$, the sum of profits increases by $p_{t}$ times

$$
\left(F\left(n+1-k_{1}, t-1\right)+F\left(n-k_{2}, t-1\right)\right)-\left(F\left(n+1-k_{2}, t-1\right)+F\left(n-k_{1}, t-1\right)\right) .
$$

This is nonnegative by (8). So the profit obtained when starting from at least one of $(n, t)$ and $(n+1, t)$ has not decreased. By definition of $k(n, t)$ the profit starting from $(n, t)$ must have strictly decreased. So the profit starting from $(n+1, t)$ must have strictly increased, which contradicts $k_{2}=k(n+1, t)$. We must therefore conclude that we cannot have $k_{2}<k_{1}$.

We now prove (8) by induction on $t$. This is clearly true when $t=0$ since then $F=0$. Suppose that $k_{0}=k(n, t)$ and $k_{2}=k(n+2, t)$. Property (C) can be proved independently of (B) (see below). So we may use (C) to observe 
that $k_{2} \leq k_{0}+2$. Then for all $j_{1} \in\{0, \ldots, n+1\}$,

$$
\begin{aligned}
F(n+2, t) & -2 F(n+1, t)+F(n, t) \\
& \leq q_{t}[F(n+2, t-1)-2 F(n+1, t-1)+F(n, t-1)] \\
& +p_{t}\left[a\left(k_{2}\right)-2 a\left(j_{1}\right)+a\left(k_{0}\right)\right. \\
& \left.+F\left(n+2-k_{2}, t-1\right)-2 F\left(n+1-j_{1}, t-1\right)+F\left(n-k_{0}, t-1\right)\right] .
\end{aligned}
$$

An application of the inductive hypothesis shows that (9) is nonpositive.

If $k_{2}=k_{0}+2$ then we can put $j_{1}=k_{0}+1$, so that (11) vanishes, and in (10) we have $a\left(k_{0}+2\right)-2 a\left(k_{0}+1\right)+a\left(k_{0}\right) \leq$ 0. If $k_{2} \leq k_{0}+1$ then in each of the middle terms in (10) and (11) that involve $j_{1}$ and have a coefficient of -2 we can once put $j_{1}=k_{0}$ and once put $j_{1}=k_{2}$. This causes (10) to vanish and (11) is nonpositive by the inductive hypothesis ( since $n+2-k_{2} \geq n+1-k_{0}$ ).

This style of proof also works to prove (B) for the groundwater management problem of (2), if we modify (8) to the statement that $F(x, t)+\Gamma(x)$ concave in $x$.

\section{A.2 Proof of $(\mathrm{A})^{*}$}

This is for the same setup as (B) above. As remarked above, we can prove a very strong form of (A), denoted (A)*. This result is not difficult, but does go beyond what has been shown by Derman, Lieberman, and Ross (1975) or Bartroff and Samuel-Cahn (2011) (who were concerned only with a continuous time model).

Let $F\left(n, p_{t-1}, \ldots, p_{1}\right)$ denote the same thing as $F(n, t)$, but let us now explicitly show the dependence on the probabilities $p_{t-1}, \ldots, p_{1}$. Similarly, let $k\left(n, p_{t-1}, \ldots, p_{1}\right)$ denote the maximizer on the right hand side of (3). We show a strong form of $(\mathrm{A})$, namely that

$$
(\mathrm{A})^{*} \quad k\left(n, p_{t-1}, \ldots, p_{1}\right) \text { is nonincreasing in each of } p_{t-1}, \ldots, p_{1} .
$$

Just as (B) is proved using (8) (concavity), so (A)* is proved by simultaneously showing that

$$
F\left(n+1, p_{t-1}, \ldots, p_{1}\right)-F\left(n, p_{t-1}, \ldots, p_{1}\right) \text { is nondecreasing in each of } p_{t-1}, \ldots, p_{1} .
$$

This is also the statement that $F(n, t)$ is supermodular in $\left(n, p_{j}\right)$ for each $j=1, \ldots, t-1$.

The proof is by induction on $t$. Note that (12)-(13) are both trivially true for $t=2$. We begin by showing an inductive step for (12). Suppose that $\left(p_{t-1}, \ldots, p_{1}\right) \leq\left(p_{t-1}^{\prime}, \ldots, p_{1}^{\prime}\right)$, componentwise. Let optimal decisions be denoted $k=k\left(n, p_{t-1}, \ldots, p_{1}\right)$ and $k^{\prime}=k\left(n, p_{t-1}^{\prime}, \ldots, p_{1}^{\prime}\right)$. Suppose that in contradiction to (12) we have $k^{\prime}>k$. Just as with the proof of (B) we consider an interchange of the usages of $k$ and $k^{\prime}$. The sum of payoffs would increase by

$$
F\left(n-k^{\prime}, p_{t-2}, \ldots, p_{1}\right)+F\left(n-k, p_{t-2}^{\prime}, \ldots, p_{1}^{\prime}\right)-F\left(n-k, p_{t-2}, \ldots, p_{1}\right)-F\left(n-k^{\prime}, p_{t-2}^{\prime}, \ldots, p_{1}^{\prime}\right)
$$

which is nonnegative assuming (13) holds with $t$ replaced by $t-1$. But use of $k$ in state $\left(n, p_{t-1}^{\prime}, \ldots, p_{1}^{\prime}\right)$ must cause a strict decrease, since $k^{\prime}$ is supposedly the least maximizer. This means that use of $k$ in state $\left(n, p_{t-1}^{\prime}, \ldots, p_{1}^{\prime}\right)$ must cause a strict increase, which is impossible by definition of $k^{\prime}$.

The proof is completed by showing an inductive step for (13). It is easy to see that the interval $[0,1]$ can be divided into subintervals such that as $p_{j}$ varies within such a subinterval the optimal policy does not change. Where such subintervals join, there is some change of policy, but at that point the value of the objective function is the same under either policy. So we may consider what happens as $p_{j}$ varies within such a subinterval. Let us begin by taking a derivative with respect to $p_{j}$, where $j \in\{1, \ldots, t-2\}$. Take $k_{1}=k(n, t)$ and $k_{2}=k(n+1, t)$. Then

$$
\begin{aligned}
\frac{d}{d p_{j}}[ & \left.F\left(n+1, p_{t-1}, \ldots, p_{1}\right)-F\left(n, p_{t-1}, \ldots, p_{1}\right)\right] \\
= & q_{t-1} \frac{d}{d p_{j}}\left[F\left(n+1, p_{t-2}, \ldots, p_{1}\right)-F\left(n, p_{t-2}, \ldots, p_{1}\right)\right] \\
& \quad+p_{t-1} \frac{d}{d p_{j}}\left[a\left(k_{2}\right)+F\left(n+1-k_{2}, p_{t-2}, \ldots, p_{1}\right)-a\left(k_{1}\right)-F\left(n-k_{1}, p_{t-2}, \ldots, p_{1}\right)\right] .
\end{aligned}
$$

We may deduce that the right hand side is nonnegative by appealing to an inductive hypothesis that (13) is true for a smaller time horizon, and the fact that (C) implies $n+1-k_{2} \geq n-k_{1}$. 
Now, let us consider taking the derivative with respect to $p_{t-1}$.

$$
\begin{aligned}
& \frac{d}{d p_{t-1}}\left[F\left(n+1, p_{t-1}, \ldots, p_{1}\right)-F\left(n, p_{t-1}, \ldots, p_{1}\right)\right] \\
& =\left[a\left(k_{2}\right)+F\left(n+1-k_{2}, p_{t-2}, \ldots, p_{1}\right)-a\left(k_{1}\right)-F\left(n-k_{1}, p_{t-2}, \ldots, p_{1}\right)\right] \\
& \quad-\left[F\left(n+1, p_{t-2}, \ldots, p_{1}\right)-F\left(n, p_{t-2}, \ldots, p_{1}\right)\right] \\
& \geq \max \left\{a\left(k_{1}+1\right)-a\left(k_{1}\right), F\left(n+1-k_{1}, p_{t-2}, \ldots, p_{1}\right)-F\left(n-k_{1}, p_{t-2}, \ldots, p_{1}\right)\right\} \\
& \quad-\left[F\left(n+1, p_{t-2}, \ldots, p_{1}\right)-F\left(n, p_{t-2}, \ldots, p_{1}\right)\right] .
\end{aligned}
$$

Consider the term in (15). We now evaluate this, conditional the event that the next meeting with the enemy occurs at time $s(\leq t-1)$, (after which there subsequently remain at most $s-1$ further meetings). Letting $j_{2}=k\left(n+1, p_{s-1}, \ldots, p_{1}\right)$ and $j_{1}=k\left(n, p_{s-1}, \ldots, p_{1}\right)$ and assuming this conditioning (which for convenience we do not try to show in any notation), we may write (15) as

$$
\begin{aligned}
& -\left[F\left(n+1, p_{t-2}, \ldots, p_{1}\right)-F\left(n, p_{t-2}, \ldots, p_{1}\right)\right] \\
& \quad=-a\left(j_{2}\right)-F\left(n+1-j_{2}, p_{s-1}, \ldots, p_{1}\right)+a\left(j_{1}\right)+F\left(n-j_{1}, p_{s-1}, \ldots, p_{1}\right) .
\end{aligned}
$$

Now by the inductive hypothesis for (12), $j_{1} \geq k_{1}$, and $j_{2} \geq k_{2}$. Also, by (B) and (C), we have $j_{1}+1 \geq j_{2} \geq j_{1}$.

In the case $j_{2}=j_{1}+1$, we deduce that (14)-(15) sum to at least

$$
a\left(k_{1}+1\right)-a\left(k_{1}\right)-\left[a\left(j_{1}+1\right)-a\left(j_{1}\right)\right] .
$$

This is nonnegative by concavity of $a(\cdot)$ and $j_{1} \geq k_{1}$.

In the case $j_{2}=j_{1}$, we deduce that (14)-(15) sum to at least

$$
\begin{aligned}
& F\left(n+1-k_{1}, p_{t-2}, \ldots, p_{1}\right)-F\left(n-k_{1}, p_{t-2}, \ldots, p_{1}\right) \\
& -\left[F\left(n+1-j_{1}, p_{s-1}, \ldots, p_{1}\right)-F\left(n-j_{1}, p_{s-1}, \ldots, p_{1}\right)\right] \\
& \geq F\left(n+1-j_{1}, p_{t-2}, \ldots, p_{1}\right)-F\left(n-j_{1}, p_{t-2}, \ldots, p_{1}\right) \\
& -\left[F\left(n+1-j_{1}, p_{s-1}, \ldots, p_{1}\right)-F\left(n-j_{1}, p_{s-1}, \ldots, p_{1}\right)\right] \\
& \geq 0,
\end{aligned}
$$

where since $j_{1} \geq k_{1}$ the first inequality follows from concavity of $F(n, \cdot)$ in $n$, and the second inequality follows from the inductive hypothesis for (12) (i.e. by putting $p_{t-1}=\cdots=p_{s}=0$ ).

\section{A.3 Proof of $(\mathrm{C})$}

The following proof is new. It is for all our models: both fighter and bomber problems. It is for the general model (3) when $a(k)$ is nondecreasing and concave in $k$, and $c(k)$ is nondecreasing and log-concave in $k$. These are more generous assumptions than those made by Bartroff and Samuel-Cahn (2011), where they give a proof of (C) for the general fighter but which only works for the particular form $c(k)=u+(1-u) a(k)$

Suppose that $k_{2}=k(n+1, t), k_{1}=k(n, t)$, but in contradiction to $(\mathrm{C})$, the resources that then remain satisfy $n-k_{1}>(n+1)-k_{2}$. This implies $k_{2}>k_{2}-1 \geq k_{1}+1>k_{1}$. The payoffs from these states, given that an investment opportunity or a meeting with the enemy occurs, are from the right hand side of (3)

$$
\begin{aligned}
P_{n+1} & =a\left(k_{2}\right)+c\left(k_{2}\right) F\left(n+1-k_{2}, t-1\right) \\
P_{n} & =a\left(k_{1}\right)+c\left(k_{1}\right) F\left(n-k_{1}, t-1\right) .
\end{aligned}
$$

To prove (C) we think about what happens if we increase $k_{1}$ to $k_{2}-1$ and decrease $k_{2}$ to $k_{1}+1$. The profits are now,

$$
\begin{aligned}
P_{n+1}^{\prime} & =a\left(k_{1}+1\right)+c\left(k_{1}+1\right) F\left(n-k_{1}, t-1\right) \\
P_{n}^{\prime} & =a\left(k_{2}-1\right)+c\left(k_{2}-1\right) F\left(n+1-k_{2}, t-1\right)
\end{aligned}
$$

Neither of these provides an improvement only if $P_{n+1} \geq P_{n+1}^{\prime}$ and $P_{n} \geq P_{n}^{\prime}$. This begs the question of whether it is possible to satisfy these two inequalities.

In the special case of $c=1$ it is easy to see that this is impossible because $P_{n+1}^{\prime}+P_{n}^{\prime}>P_{n+1}+P_{n}$.

In the special case of $c=a$ it is easy to see that this is impossible because $P_{n+1}^{\prime} P_{n}^{\prime}>P_{n+1} P_{n}$. 
So it is easy to prove $(\mathrm{C})$ for the invincible or frail fighter. It is more difficult to see what happens for more general choices of $a$ and $c$. Let $x=F\left(n+1-k_{2}, t-1\right)$ and $y=F\left(n-k_{1}, t-1\right)$. Now

$$
\begin{aligned}
P_{n+1} \geq P_{n+1}^{\prime} & \Longleftrightarrow a\left(k_{2}\right)+c\left(k_{2}\right) x \geq a\left(k_{1}+1\right)+c\left(k_{1}+1\right) y \\
P_{n} \geq P_{n}^{\prime} & \Longleftrightarrow a\left(k_{1}\right)+c\left(k_{1}\right) y \geq a\left(k_{2}-1\right)+c\left(k_{2}-1\right) x .
\end{aligned}
$$

By concavity of $a(\cdot),(16)$ implies that we must have

$$
a\left(k_{2}-1\right)+a\left(k_{1}+1\right)-a\left(k_{1}\right)+c\left(k_{2}\right) x \geq a\left(k_{1}+1\right)+c\left(k_{1}+1\right) y .
$$

So putting $d=a\left(k_{2}-1\right)-a\left(k_{1}\right) \geq 0$ we have that (16)-(17) imply

$$
\begin{array}{r}
d+c\left(k_{2}\right) x-c\left(k_{1}+1\right) y \geq 0 \\
-d+c\left(k_{1}\right) y-c\left(k_{2}-1\right) x \geq 0 .
\end{array}
$$

Using $\log$-concavity of $c$, so that $c\left(k_{1}\right) \leq c\left(k_{2}-1\right) c(k-1+1) / c\left(k_{2}\right)$, we find that (19) requires

$$
-c\left(k_{2}\right) d+c\left(k_{2}-1\right)\left[c\left(k_{1}+1\right) y-c\left(k_{2}\right) x\right] \geq 0 .
$$

The term in square brackets must be nonnegative, and so since $c\left(k_{2}\right) \geq c\left(k_{2}-1\right)$, this implies that we must have

$$
-d+c\left(k_{1}+1\right) y-c\left(k_{2}\right) x \geq 0 .
$$

The inequalities above become strict if the functions $a$ and $c$ are not constant functions. When inequalities are strict (20) is in contradiction to (18). Therefore we may conclude that at least one of the changes $k_{1} \rightarrow k_{2}-1$ and $k_{2} \rightarrow k_{1}+1$ makes a strict improvement, and that so the posited counterexample to (C) cannot occur.

\section{B Proof of (A) for a special case of general fighter}

Consider the a special case of the fighter problem defined by (3), but with $p_{t}=1$. So the dynamic programming equation is

$$
F(n, t)=\max _{k \in\{1, \ldots, n\}}[a(k)+c(k) F(n-k, t-1)],
$$

with $F(n, 0)=0$. In other words, we wish to maximize

$$
a\left(k_{1}\right)+c\left(k_{1}\right) a\left(k_{2}\right)+c\left(k_{1}\right) c\left(k_{2}\right) a\left(k_{3}\right)+\cdots+c\left(k_{1}\right) c\left(k_{2}\right) \cdots c\left(k_{t-1}\right) a\left(k_{t}\right),
$$

subject to $k_{1}+\cdots+k_{t}=n$. Here $k_{i}$ is the units of ammunition to be used at the $i$ th meeting with the enemy. For the optimal values of these variables we might write $k_{i}^{*}$, but for convenience we omit the stars in what follows.

Our aim is to show (A), namely that for any fixed $n$, the optimal $k_{1}$ is a nonincreasing function of $t$. In the case that $0<u<1$ and $c(k)=u+(1-u) a(k)$, we can write $a(k)=(c(k)-u) /(1-u)$, substitute, and write our objective function as

$$
-\frac{u}{1-u}+c\left(k_{1}\right)+c\left(k_{1}\right) c\left(k_{2}\right)+\cdots+c\left(k_{1}\right) \cdots c\left(k_{t-1}\right)+\frac{1}{1-u} c\left(k_{1}\right) \cdots c\left(k_{t}\right) .
$$

But this is of no obvious assistance in showing that $k_{1}$ should be nondecreasing in $t$.

Let us remark that it is trivial to establish (B) for the bomber problem when $p=1$ and $c(k)$ is log-concave (the objective being to maximize $\left.c\left(k_{1}\right) \cdots c\left(k_{t}\right)\right)$. We should take $k_{1}=\lfloor n / t\rfloor$ (or $k_{1}=\lceil n / t\rceil$ ), which is trivially nondecreasing in $n$.

Can we prove that (A) holds for the general fighter problem when $p=1$ and $a(k)$ is concave, and $c(k)=$ $u+(1-u) a(k)$ ? It is only for the invincible fighter $(u=1)$ that $(\mathrm{A})$ is obvious, since then the objective is $a\left(k_{1}\right)+\cdots+a\left(k_{t}\right)$ and we should take $k_{1}=\lfloor n / t\rfloor$. For the general fighter there is no optimal solution that we can simply write down, such as $k_{1}=\lfloor n / t\rfloor$. We must argue in a more cunning way. In cases of the invincible and frail fighters, the proof of (A) is via showing supermodularity (also sometimes called 'increasing differences'), in $(n, t)$, of either $F(n, t)$ or $\log F(n, t)$, respectively. The following proof is of a non-traditional form, because it does not involve this sort of inductive proof of some property of the optimal value function.

Let us consider the problem of maximizing

$$
a\left(k_{1}\right)+c\left(k_{1}\right) a\left(k_{2}\right)+c\left(k_{1}\right) c\left(k_{2}\right) a\left(k_{3}\right)+\cdots+c\left(k_{1}\right) c\left(k_{2}\right) \cdots c\left(k_{t-1}\right) a\left(k_{t}\right) X,
$$

subject to $k_{1}+\cdots+k_{t}=n$, where $X$ is a nonnegative quantity that we allow to vary. Denote the maximized value as $F_{X}(n, t)$. 
We will show that $k_{1}$ is a nonincreasing function of $t$ by showing that $k_{1}$ is a nonincreasing function of $X$. We then apply this result as $X$ increases from 0 to 1 , i.e. as we increase the number of enemy encounters from $t-1$ to $t$.

First, notice that it is easy to show that $k_{1} \geq k_{2} \geq \cdots \geq k_{t}$ (by a simple interchange argument).

Imagine increasing $X$ continuously from 0 . The optimal $k_{i}$ will change at various points. Consider any value of $X$, say $X=x$, at which it becomes optimal to change some of the $k_{i}$ values. Call the new values, $k_{1}^{\prime}, \ldots, k_{t}^{\prime}$ (these being optimal at $X=x$ and at values of $X$ just greater than $x$ ). Suppose that $k_{1}^{\prime}>k_{1}$ (in contradiction to (A)). Then by property (C) (which one can show for this problem in the usual way) we must have

$$
\begin{gathered}
k_{1}^{\prime}>k_{1} \\
k_{1}^{\prime}+k_{2}^{\prime} \geq k_{1}+k_{2} \\
k_{1}^{\prime}+k_{2}^{\prime}+k_{3}^{\prime} \geq k_{1}+k_{2}+k_{3} \\
\vdots \quad \vdots \\
k_{1}^{\prime}+k_{2}^{\prime}+\cdots+k_{t}^{\prime}=k_{1}+k_{2}+\cdots+k_{t} .
\end{gathered}
$$

Now $F_{X}(n, t)$ is a convex function of $X$ (being the maximum of linear functions of $X$ ). So as the $k_{i}$ change to $k_{i}^{\prime}$ we must see that the coefficient of $X$ is nondecreasing. This coefficient is

$$
c\left(k_{1}\right) c\left(k_{2}\right) \cdots c\left(k_{t-1}\right) a\left(k_{t}\right)=c\left(k_{1}\right) c\left(k_{2}\right) \cdots c\left(k_{t-1}\right) c\left(k_{t}\right) \frac{a\left(k_{t}\right)}{c\left(k_{t}\right)} .
$$

Notice that $k_{t}^{\prime} \leq k_{t}$. Taking the canonical specification of $a(k)$ being concave and $c(k)=u+(1-u) a(k)$, it is easy to check that $a\left(k_{t}^{\prime}\right) / c\left(k_{t}^{\prime}\right) \leq a\left(k_{t}\right) / c\left(k_{t}\right)$. However, the product $c\left(k_{1}\right) c\left(k_{2}\right) \cdots c\left(k_{t-1}\right) c\left(k_{t}\right)$ also decreases as we change $k_{i}$ to $k_{i}^{\prime}$. This follows from the Karamata Majorization Inequality, which says as follows.

Karamata Majorization Inequality Suppose $x$ and $y$ are vectors with $x_{1} \geq \cdots \geq x_{t}$ and $y_{1} \geq \cdots \geq y_{t}$ and $x$ majorizes $y$ (written $x \succ y$ ), in the sense that $\sum_{i=1}^{j} x_{i} \geq \sum_{i=1}^{j} y_{i}$ for all $j=1, \ldots, t-1$, and $\sum_{i=1}^{t} x_{i}=\sum_{i=1}^{t} y_{i}$. Suppose $f$ is a concave function. Then

$$
\sum_{i=1}^{t} f\left(x_{i}\right) \leq \sum_{i=1}^{t} f\left(y_{i}\right)
$$

See Marshall and Olkin (1979) for a proof.

We apply this inequality with $f(k)=\log c(k)$ (which is a strictly concave function of $m$ ), to deduce that if $k_{1}^{\prime}>k_{1}$ then $m^{\prime} \succ m$ (as argued above) and hence

$$
c\left(k_{1}^{\prime}\right) \cdots c\left(k_{t}^{\prime}\right)<c\left(k_{1}\right) \cdots c\left(k_{t}\right)
$$

and we conclude

$$
c\left(k_{1}^{\prime}\right) \cdots c\left(k_{t-1}^{\prime}\right) a\left(k_{t}^{\prime}\right)<c\left(k_{1}\right) \cdots c\left(k_{t-1}\right) a\left(k_{t}\right) .
$$

This is inconsistent with the fact that $F_{X}(n, t)$ is convex in $X$. We must therefore conclude that $k_{1}$ is nonincreasing in $X$.

The above proof seems rather a heavy-handed for a result that is so obvious. I would be interested if a reader could prove this result in a simpler way.

\section{Small results about (B) in the bomber problem}

Consider the discrete-time discrete-ammunition bomber problem. It is as good to spend all units of the remaining resource as it is to spend all but 1 unit if

$$
c(n) F(0, t-1) \geq c(n-1) F(1, t-1),
$$

where $F(0, t)$ and $F(1, t)$ are solutions to

$$
\begin{aligned}
& F(0, t)=q F(0, t-1)+p c(0) F(0, t-1), \\
& F(1, t)=q F(1, t-1)+p c(1) F(0, t-1),
\end{aligned}
$$

with $F(0,0)=F(1,0)=1$. Upon solving these recurrences, we find that (21) is equivalent to

$$
\frac{c(n)}{c(n-1)} \geq \frac{1}{c(0)}\left[c(1)-(c(1)-c(0))\left(\frac{q}{q+p c(0)}\right)^{t-1}\right] \text {. }
$$


In the limit $c(0) \rightarrow 0$ this becomes

$$
\frac{c(n)}{c(n-1)} \geq 1+(p / q)(t-1) c(1) .
$$

Notice that if (22) (or (23)) is satisfied, then it remains satisfied if either $n$ or $t$ decreases. Thus, we may deduce the following lemma directly from the dynamic programming equation.

Lemma 1 If (22) holds then $k(n, t)=n$, and

$$
F(n, t)=q^{t}+\frac{c(n)}{c(0)}\left((q+p c(0))^{t}-q^{t}\right) .
$$

In the case $c(0)=0$, similarly (23) implies $k(n, t)=n$ and $F(n, t)=q^{t}+t p q^{t-1} c(n)$.

By taking $p=T / t, n=x N$ and $c(n)=1-u e^{-n / N}$, then letting both $N$ and $t$ tend to infinity one can recover some of the same results that have been obtained by Bartroff (2011) for the case of both continuous ammunition and time.

\section{Lemma 2}

(i) $k(n+1, t) \geq k(n, t)$ for all $n$ and $t=1,2,3$.

(ii) If $c(k)$ is concave in $k$ then $F(n, 1)=1-p+p c(n)$ is concave in $n$.

(iii) If $c(k)=1-\theta^{k}$, then, with $q_{i}=1-p_{i}$, then

$$
F(n, 2)=q_{1} q_{2}+\max _{k \in\{1, \ldots, n\}}\left\{q_{1} p_{2} c(n)+p_{1} q_{2} c(k)+p_{1} p_{2} c(k) c(n-k)\right\}
$$

is log-concave in $n$.

Statement (i) follows from (ii) and (iii). It is only (iii) that is difficult to prove. I know only a rather long and tedious proof, making use of Mathematica to assist with the algebra, and which requires one to assume the special form of $c(k)=1-\theta^{k}$. I omit a proof from this paper and leave it as a challenge to some interested reader to prove (iii) in a better way. Counterexample 2 tells us that (iii) is not true if $c(\cdot)$ is simply assumed to be concave.

Lemma $3 k(n, t)=1 \Longrightarrow k(n-1, t)=1$.

Proof This follows from a fact that we have already mentioned in Section 3 . This is that $k(n-k(n, t), t-1) \leq k(n, t)$. To see this, notice that if $k(n-k(n, t), t-1)>k(n, t)$ then by (A) we must have $k(n-k(n, t), s)>k(n, t)$ for all $s \leq t-1$. One can check that a better policy is obtained by increasing $k(n, t)$ by 1 and decreasing every $k(n-k(n, t), s)$ by 1 .

A corollary of Lemma 3 is the following.

Theorem 1 If we are constrained to firing only 1 or 2 units of ammunition at each meeting with the enemy then (B) is true.

By using Lemmas 2 and 3 one can fairly easily establish the fact that (B) is true when either $t$ or $n$ is small.

Theorem 2 If $n \leq 3$ or $t \leq 3$ then $k(n+1, t) \geq k(n, t)$. 